\title{
Macrophages contribute to liver repair after monocrotaline-induced liver injury via SDF-1/CXCR4
}

\author{
FUMISATO OTAKA $^{1-3}$, YOSHIYA ITO ${ }^{1,2}$, SHUJI NAKAMOTO ${ }^{1,4}$, NOBUYUKI NISHIZAWA ${ }^{1,4}$, \\ TETSUYA HYODO $^{1,5}$, KANAKO HOSONO ${ }^{1,2}$, MASATAKA MAJIMA ${ }^{2,6}$, \\ WASABURO KOIZUMI ${ }^{3}$ and HIDEKI AMANO ${ }^{1,2}$ \\ ${ }^{1}$ Department of Molecular Pharmacology, Graduate School of Medical Sciences, Kitasato University; \\ Departments of ${ }^{2}$ Pharmacology, ${ }^{3}$ Gastroenterology, ${ }^{4}$ Surgery and ${ }^{5}$ Plastic Surgery, Kitasato University \\ School of Medicine, Sagamihara, Kanagawa 252-0374; ${ }^{6}$ Department of Medical Therapeutics, \\ Kanagawa Institute of Technology, Atsugi, Kanagawa 243-0292, Japan
}

Received November 27, 2020; Accepted February 18, 2021

DOI: $10.3892 / \mathrm{etm} .2021 .10100$

\begin{abstract}
Monocrotaline (MCT) administration induces liver injury in rodents that mimics the pathology of human sinusoidal obstruction syndrome. MCT-induced SOS models are used to investigate the mechanism of injury and optimize treatment strategies. However, the processes underlying liver repair are largely unknown. Specifically, the role of macrophages, the key drivers of liver repair, has not been elucidated. The current study aimed to examine the role of macrophages in the repair of MCT-induced liver injury in male C57/BL6 mice. Maximal liver injury occurred at $48 \mathrm{~h}$ post-MCT treatment, followed by repair at $120 \mathrm{~h}$ post-treatment. Immunofluorescence analysis revealed that $\mathrm{CD} 68^{+}$macrophages were recruited to the injured regions after MCT treatment. This was associated with the decreased expression of genes related to a pro-inflammatory macrophage phenotype and the increased expression of those associated with a reparative macrophage phenotype during the repair phase. The results also revealed that stromal cellderived factor-1 (SDF-1) and its receptor C-X-C chemokine receptor-4 (CXCR4) were upregulated, and $\mathrm{CD}^{+} 8^{+}$macro-
\end{abstract}

Correspondence to: Dr Yoshiya Ito, Department of Pharmacology, Kitasato University School of Medicine, 1-15-1 Kitasato, Minami-ku, Sagamihara, Kanagawa 252-0374, Japan

E-mail: yito@kitasto-u.ac.jp

Abbreviations: ALT, alanine transaminase; ALI, acute liver injury; $\mathrm{BM}$, bone marrow; CCR2, C-C motif chemokine receptor 2; CXCR4, $\mathrm{C}-\mathrm{X}-\mathrm{C}$ chemokine receptor type 4; EGF, epidermal growth factor; GFP, green fluorescent protein; H\&E, hematoxylin and eosin; HGF, hepatocyte growth factor; IL, interleukin; i.p., intraperitoneally; I/R, ischemia reperfusion; LSEC, liver sinusoidal endothelial cell; MCT, monocrotaline; MR, mannose receptor; SD, standard deviation; SDF-1, stromal cell-derived factor 1; SOS, sinusoidal obstruction syndrome

Key words: liver repair, monocrotaline, macrophage, stromal cellderived factor $1, \mathrm{C}-\mathrm{X}-\mathrm{C}$ chemokine receptor type 4 phages were co-localized with CXCR4 expression. Treatment of mice with AMD3100, a CXCR4 antagonist, delayed liver repair and increased the expression of genes related to a proinflammatory macrophage phenotype. In contrast, SDF-1 treatment stimulated liver repair and increased the expression of genes related to a reparative macrophage phenotype. The results suggested that macrophages accumulate in the liver and repair damaged tissue after MCT treatment, and that the SDF-1-CXCR4 axis is involved in this process.

\section{Introduction}

Sinusoidal obstruction syndrome (SOS) is a progressive and potentially fatal complication of radiotherapy in patients preparing for hematopoietic stem cell transplantation and chemotherapy for liver metastasis of colorectal cancer (1-3). Severe SOS has a high mortality rate. SOS is considered to be related to radiation- or chemotherapy-induced damage to the liver microvasculature $(1,4)$. Toxic doses of monocrotaline (MCT), a pyrrolizidine alkaloid present in plants of genus Crotalaria, have been used to induce SOS in rats for use as experimental models to study liver injury in vivo. MCT-induced liver injury is characterized by the formation of a gap at the surface of liver sinusoidal endothelial cells (LSECs), which leads to sinusoidal hemorrhage and centrilobular hepatocellular necrosis. Sinusoidal destruction is accompanied by infiltration of the centrilobular regions by inflammatory cells $(4,5)$. Once the liver is injured, it must be repaired and regenerated. Indeed, rats that survive have shown resolution of MCT-induced liver inflammation $(4,5)$. Furthermore, hepatic tissue repair plays a critical role in determining the final outcome of chemical-induced hepatotoxicity (6). However, the process of liver repair during MCT hepatotoxicity and its underlying mechanism is largely unknown.

Macrophages play a critical role in liver repair following acute injury induced by chemicals and ischemia/reperfusion (I/R) (7-11). Recently, we showed that MCT-induced liver injury is associated with accumulation of macrophages (12). Typically, accumulation of macrophages at the site of injury promotes the recovery of damaged tissues. However, it is 
unclear whether accumulated macrophages play a role in liver repair after MCT-induced liver injury.

$\mathrm{C}-\mathrm{X}-\mathrm{C}$ chemokine receptor type 4 (CXCR4) is a receptor for stromal cell-derived factor 1 (SDF-1) (13). CXCR4 signaling plays a crucial role in the mobilization and recruitment of progenitor cells from the bone marrow (BM), which stimulates angiogenesis and recovery of ischemic tissue $(13,14)$. In addition, SDF-1-CXCR4 signaling contributes to tissue repair after acute limb ischemia (15) and acute gastric ulcers (16) in mice by recruiting pro-angiogenic macrophages from the BM. Accumulating evidence suggests that SDF-1-CXCR4 is involved in liver repair after acute injury $(13,17)$. CXCR4 blockade inhibits hepatocyte proliferation in mice treated with acetaminophen, indicating that CXCR4 signaling promotes liver regeneration (18). Recruitment of CXCR4-expressing hematopoietic progenitors in response to SDF-1 is an important mechanism underlying the repair of liver tissue (19); of note, these findings indicate that the SDF-1-CXCR4 signaling pathway promotes tissue recovery from ischemia- or chemical-induced injury via the accumulation of pro-angiogenic macrophages. In addition, CXCR4 also plays a role in the development of MCT-induced pulmonary arterial hypertension and vascular remodeling (20). Altogether, these above findings led us to investigate whether the SDF-1-CXCR4 axis contributes to liver repair in the context of MCT-induced liver injury through the recruitment of macrophages.

Here, we investigated the role of macrophages in liver repair after MCT-induced liver injury. Further, we examined whether SDF-1-CXCR4 axis contributes to macrophage accumulation and tissue repair in mice after MCT-induced hepatotoxicity.

\section{Materials and methods}

Animals. Male C57BL/6 WT mice (8-10-weeks-old) were purchased from CLEA Japan. Transgenic mice expressing green fluorescent protein (GFP) against a C57BL/6 background were kindly provided by Dr Okabe (Genome Information Research Center, Osaka University, Osaka, Japan). Mice were maintained on a $12 \mathrm{~h}$ light/dark cycle in a facility with constant humidity $(50 \% \pm 5 \%)$ and temperature $\left(25 \pm 1^{\circ} \mathrm{C}\right)$, and were provided with food and water ad libitum. All experimental procedures were approved by the Animal Experimentation and Ethics Committee of the Kitasato University School of Medicine (2019-036, 2020-103), and were performed in following the guidelines for animal experiments set down by the Kitasato University School of Medicine, which are in accordance with the 'Guidelines for Proper Conduct of Animal Experiments' published by the Science Council of Japan.

Animal procedures. Animals were fasted overnight and then injected intraperitoneally (i.p.) with $600 \mathrm{mg} / \mathrm{kg}$ MCT (Merck $\mathrm{KGaA}$ ) dissolved in warm pyrogen-free saline (final concentration, $2.0 \mathrm{mg} / \mathrm{ml}$ ) to induce SOS (12). A total of 70 mice were used in this study. Mice were anesthetized with pentobarbital sodium (60 mg/kg i.p.) at $0(n=8), 24(n=8), 48(n=8), 72$ $(\mathrm{n}=7), 96(\mathrm{n}=8)$, and $120 \mathrm{~h}(\mathrm{n}=8)$ after MCT administration; approximately $500 \mu \mathrm{l}$ of blood were collected from the heart of each mice. The levels of alanine transaminase (ALT) were measured using a Dri-Chem 7000 Chemistry Analyzer System (FujiFilm). Immediately after blood collection, the livers were excised and rinsed in saline. A small section of each liver was placed in $10 \%$ formaldehyde, and the remaining liver was frozen in liquid nitrogen and stored at $-80^{\circ} \mathrm{C}$ for further analysis. Afterwards, the animals were euthanized by cervical dislocation, and the death was verified by the lack of heartbeat, respiration and corneal reflex.

Mice received a daily i.p. injection of a CXCR4 antagonist (AMD3100; 10 mg/kg; Sigma-Aldrich; Merck KGaA) (n=4) in $100 \mu 1$ phosphate-buffered saline (PBS) (21) or vehicle $(n=5)$. A group of mice received a daily i.p. injection of recombinant murine SDF-1 $\alpha(20 \mu \mathrm{g} / \mathrm{kg}, \mathrm{R} \& \mathrm{D}$ Systems Inc.) (n=5) (22) or vehicle $(n=4)$. An identical volume of sterile PBS was used as the vehicle control. At $72 \mathrm{~h}$, mice were anesthetized with pentobarbital sodium (60 mg/kg i.p.), and the blood and liver samples were collected. These mice were euthanized by cervical dislocation.

Histology and immunohistochemistry. Excised liver tissues were fixed immediately with $10 \%$ formaldehyde prepared in $0.1 \mathrm{M}$ sodium phosphate buffer ( $\mathrm{pH} 7.4)$. Sections $(4 \mu \mathrm{m})$ were prepared from paraffin-embedded tissues and stained with hematoxylin and eosin (H\&E). Images of H\&E-stained sections were captured under a microscope (Biozero BZ-9000 Series; Keyence Corporation).

Immunofluorescence analysis. Fixed liver samples were embedded in Tissue-Tek O.C.T. Compound (Sakura Finetek USA, Inc.), frozen at $-80^{\circ} \mathrm{C}$, and cut into $8 \mu \mathrm{m}$ sections using a cryostat. The sections were incubated overnight at $4{ }^{\circ} \mathrm{C}$ with a rat anti-mouse CD68 monoclonal antibody (Bio-Rad Laboratories, Inc.), Cy3-labeled mouse anti- $\alpha$ smooth muscle actin ( $\alpha$ SMA) (Sigma-Aldrich; Merck KGaA), a rat anti-mouse CXCR4 monoclonal antibody (Invitrogen; Thermo Fisher Scientific, Inc.), or rabbit anti-mouse SDF-1 polyclonal antibody (Abcam). After washing with PBS, the sections were incubated for $1 \mathrm{~h}$ at room temperature with Alexa Fluor 488-conjugated donkey anti-rabbit IgG and Alexa Fluor 594-conjugated donkey anti-rat IgG (Molecular Probes; Thermo Fisher Scientific, Inc.). Stained sections were observed under a fluorescence microscope (Biozero BZ-9000; Keyence Corporation) and images were captured. Expression of CD68 in the liver tissues from ten fields per section at x400 magnification was measured as fluorescence intensity using ImageJ software, version 1.50i (National Institutes of Health). The results were expressed as the average of CD68 fluorescence intensity per field.

Quantitative real-time RT-PCR. Total RNA was extracted from mouse tissues and homogenized in TRIzol Reagent (Life Technologies; Thermo Fisher Scientific, Inc.). Singlestranded cDNA was generated from $1 \mu \mathrm{g}$ of total RNA by reverse transcription using a ReverTra Ace qPCR RT kit (Toyobo Co., Ltd.), according to the manufacturer's instructions. Quantitative PCR was performed using TB Green Premix Ex Taq II (Tli RNaseH Plus; Takara Bio, Inc.). Genespecific primers used for real-time RT-PCR were designed using Primer 3 software (http://primer3.sourceforge.net/), based on data from GenBank. The primer sequences are listed in Table I. Data were normalized to the expression of glyceraldehyde-3-phosphate dehydrogenase in each sample. 
Table I. Primers used for reverse transcription and quantitative PCR reactions.

\begin{tabular}{lll}
\hline Gene & Forward primer sequence $\left(5^{\prime}-3^{\prime}\right)$ & Reverse primer sequence $\left(5^{\prime}-3^{\prime}\right)$ \\
\hline$H G F$ & GGCTGAAAAGATTGGATCAGG & CCAGGAACAATGACACCAAGA \\
$E G F$ & ATGGGAAACAATGTCACGAAC & CATCTCTCCCAAGCACTGAAC \\
$T N F \alpha$ & TCTTCTCATTCCTGCTTGTGG & GATCTGAGTGTGAGGGTCTGG \\
$I L-1 \beta$ & TACATCAGCACCTCACAAGCA & CCAGCCCATACTTTAGGAAGA \\
$F i z z l$ & CAAAGCCAGAGTCCTTCAGAG & TAGGAGAGCATTGGAAATTGG \\
$M R$ & TGCCAATCCAGCTAACTATCC & CACACCCAGTAGCAGTCATCC \\
$S D F-1$ & TTTGTCCATTGCACTTTGAGG \\
$C C R 2$ & GCATCAGTGACGGTAAACCAG & TGCCAGGTAAAGCAGACTTG \\
$G a p d h$ & CTCTGAAGAAGTGGGGTCTGG & GCACAGTTGGAGTGTTGAGG \\
\hline
\end{tabular}
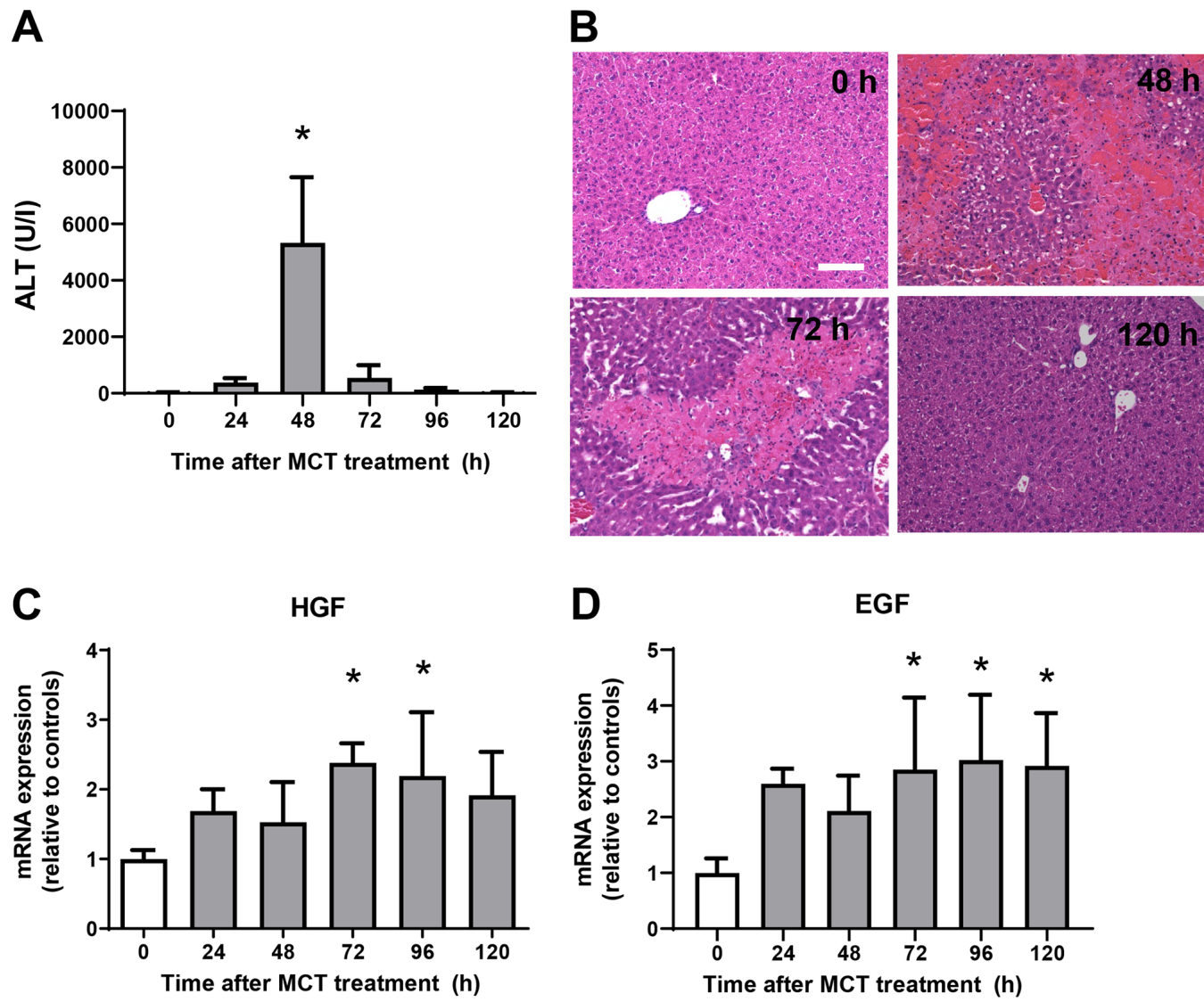

Figure 1. Liver injury after monocrotaline (MCT) treatment. (A) Time-dependent changes in ALT levels during MCT hepatotoxicity (n=7-8 mice per group). (B) Typical histological appearance of hematoxylin and eosin-stained liver sections were analyzed at $0,48,72$ and $120 \mathrm{~h}$ post-MCT treatment (scale bars, $100 \mu \mathrm{m}$ ). Expression of (C) HGF and (D) EGF mRNA in the livers of mice treated with MCT. Data are presented as the mean \pm SD ( $\mathrm{n}=5-6$ mice per group). ${ }^{*} \mathrm{P}<0.05$ vs. 0 h. MCT, monocrotaline; ALT, alanine transaminase; HGF, hepatocyte growth factor; EGF, epidermal growth factor.

BM transplantation. BM transplantation was performed as previously described (10). Briefly, recipient mice $(n=3)$ were treated with clodronate-loaded liposomes (200 $\mu \mathrm{l} / \mathrm{mouse}$; FormuMax Scientific, Inc.) to deplete the tissue macrophages $48 \mathrm{~h}$ before irradiation. Following euthanasia via cervical dislocation under $4 \%$ isoflurane anesthesia, donor BM cells were harvested from male $\mathrm{GFP}^{+}$transgenic mice ( 8 weeks old) $(n=1)$. Mice were irradiated with 9.8 Gy using an MBR-1505R $\mathrm{X}$-ray irradiator (Hitachi Medical Co.) fitted with a filter (copper, $0.5 \mathrm{~mm}$; aluminum, $2 \mathrm{~mm}$ ); the cumulative radiation dose was monitored throughout. Irradiated mice received donor BM-derived mononuclear cells $\left(1 \times 10^{7}\right.$ cells/200 $\left.\mu 1 \mathrm{PBS}\right)$ via injection in the tail vein. Following euthanasia via cervical dislocation under $4 \%$ isoflurane anesthesia, liver tissues were also collected.

Statistical analysis. All results are presented as mean \pm standard deviation (SD). All statistical analyses were performed 


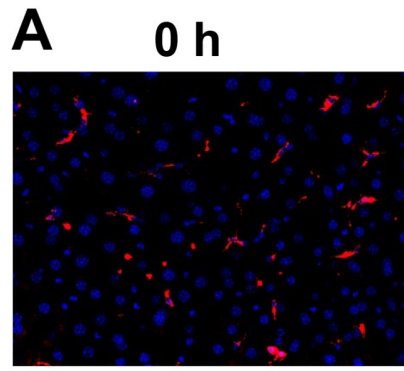

$72 \mathrm{~h}$
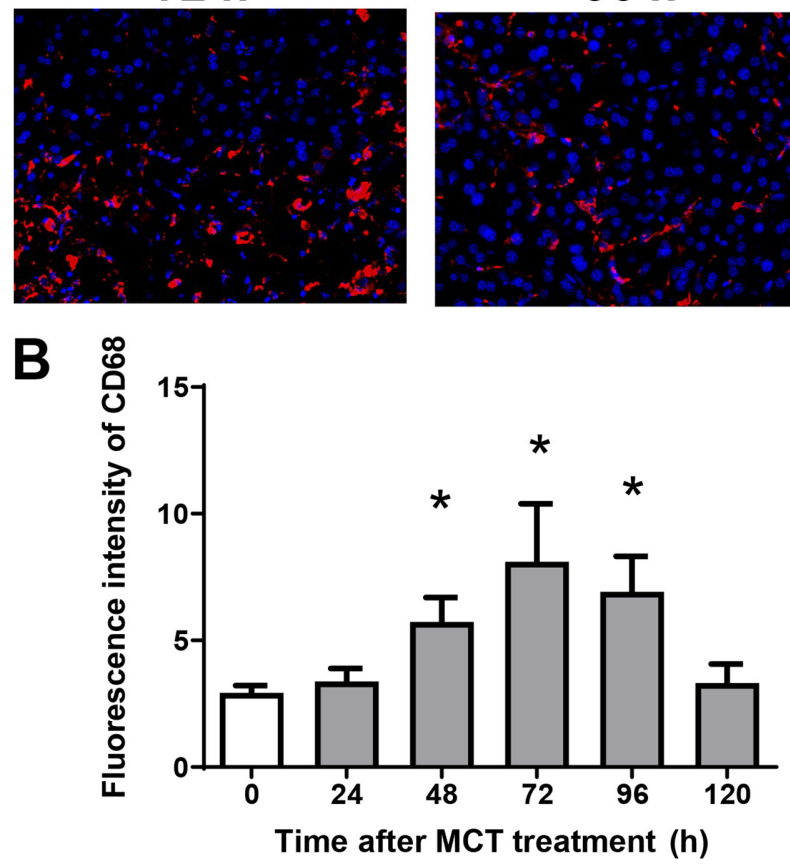

$24 \mathrm{~h}$

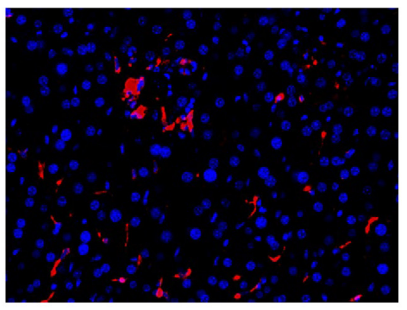

$96 \mathrm{~h}$
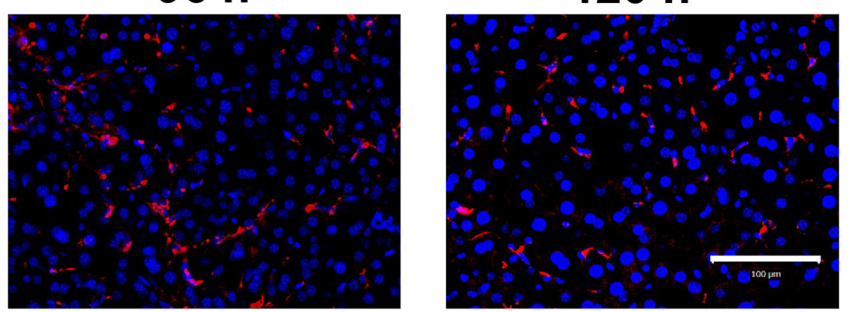

$48 \mathrm{~h}$

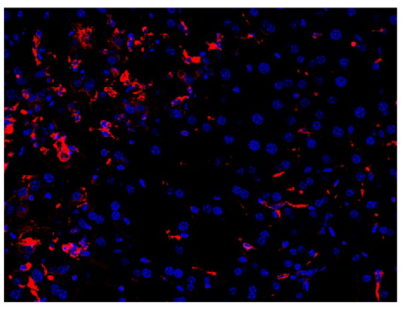

$120 \mathrm{~h}$ Figure 2. Accumulation of macrophages during MCT hepatotoxicity. (A) Immunofluorescence analysis of the macrophage marker CD68 (red) in the livers of
mice treated with MCT. Nuclei are stained with DAPI (blue). Scale bars represent $100 \mu \mathrm{m}$. (B) Fluorescence intensity of CD68 in the livers of mice treated with MCT. Data are expressed as the mean $\pm \mathrm{SD}$ ( $\mathrm{n}=4$ mice per group). ${ }^{*} \mathrm{P}<0.05$ vs. $0 \mathrm{~h}$. MCT, monocrotaline.

using GraphPad Prism software, version 8 (GraphPad Software). Data from two groups were compared using an unpaired two-tailed Student's t-test, and data from multiple groups were compared using one-way analysis of variance followed by Tukey's post-hoc test. A P-value $<0.05$ was considered statistically significant.

\section{Results}

MCT-induced liver injury and liver repair. ALT levels were measured from $0 \mathrm{~h}$ to $120 \mathrm{~h}$ after MCT administration (Fig. 1A). ALT levels increased significantly, reaching maximal levels at $48 \mathrm{~h}$ post-MCT treatment before falling again and returning to normal levels at $120 \mathrm{~h}$ post-treatment. Histological analyses of the liver demonstrated minimal changes at $0 \mathrm{~h}$ after MCT treatment (Fig. 1B). At $48 \mathrm{~h}$ post-MCT treatment, significant hemorrhagic necrosis in the centrilobular regions of the liver was observed. At $72 \mathrm{~h}$ post-MCT treatment, hepatic necrosis around the central veins was clearly localized and the necrotic area was reduced, which was accompanied by cellular infiltration of the injured regions. Hepatic necrosis was diminished and changes were much less obvious at $120 \mathrm{~h}$ post-MCT treatment. These results suggest that MCT-induced liver injury peaks at $48 \mathrm{~h}$, followed by liver repair from $72 \mathrm{~h}$ and resolution at $120 \mathrm{~h}$ post-MCT treatment. We also measured the expression of mRNA encoding tissue repair factors, hepatocyte growth factor (HGF) and epidermal growth factor (EGF) (Fig. 1C and D) (11). Expression levels of HGF and EGF mRNA increased during the repair phase (from $72 \mathrm{~h}$ to $120 \mathrm{~h}$ after MCT treatment). These findings suggest that the liver recovered from MCT-induced liver injury, as demonstrated by reduced ALT levels, diminished area of hepatic necrosis, and increased expression of growth factors.

Accumulation of macrophages during MCT hepatotoxicity. Because hepatic macrophages play an important role in stimulating liver repair after acute liver injury (ALI), we next examined the effects of MCT on the accumulation of macrophages in the liver. Immunofluorescence analysis revealed that $\mathrm{CD}^{+} 8^{+}$cells (macrophages) accumulated in the liver after MCT treatment (Fig. 2A). The fluorescence intensity of hepatic CD68 increased, peaking at $72 \mathrm{~h}$ after MCT treatment (Fig. 2B). These results suggest that accumulation of $\mathrm{CD} 68^{+}$ cells is associated with liver repair after MCT-induced ALI.

Expression of mRNA encoding genes associated with a pro-inflammatory phenotype and a reparative macrophage phenotype. Next, we examined the expression of mRNA 

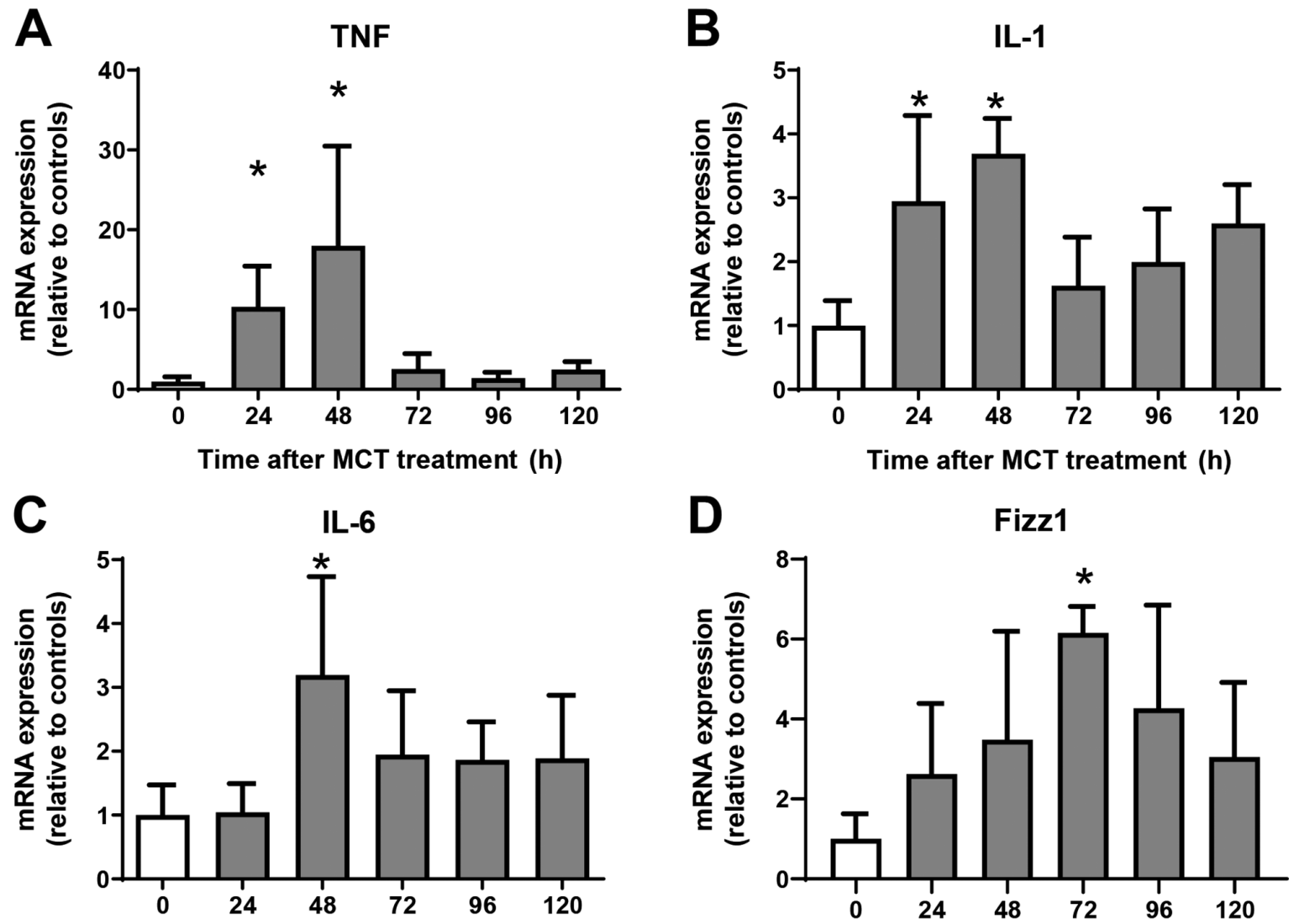

Time after MCT treatment (h)

Time after MCT treatment (h)

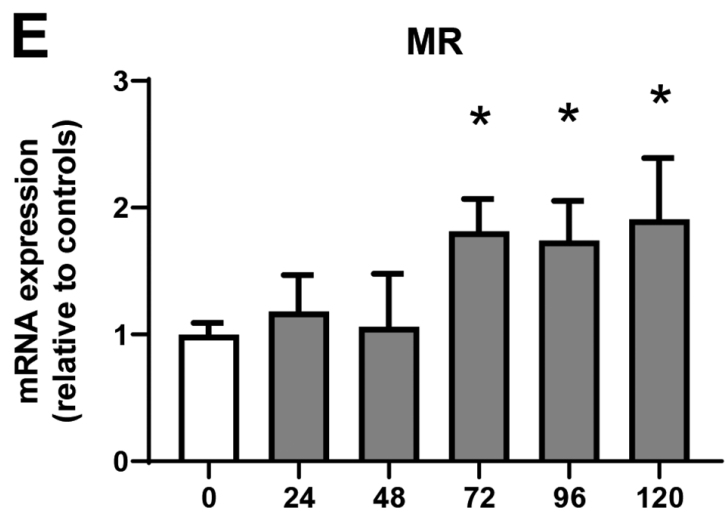

Time after MCT treatment (h)

Figure 3. Changes in mRNA expression of encoding genes associated with a pro-inflammatory macrophage phenotype and a reparative macrophage phenotype during hepatotoxicity. mRNA expression of pro-inflammatory macrophage (A) TNF $\alpha$, (B) IL-1 $\beta$ and (C) IL-6, and markers of reparative macrophages, including (D) Fizz1 and (E) mannose receptor in the livers of mice treated with MCT. Data are expressed as the mean \pm SD (n=4-6 mice per group). ${ }^{*} \mathrm{P}<0.05$ vs. 0 h. MCT, monocrotaline; TNF, tumor necrosis factor; IL, interleukin; Fizz1, found in inflammatory zone 1; MR, mannose receptor.

encoding genes of a pro-inflammatory macrophage phenotype, tumor necrosis factor $\alpha(\mathrm{TNF} \alpha)$, interleukin (IL)-1 $\beta$, and IL-6, and of a reparative macrophage phenotype, found in inflammatory zone 1 (Fizz1) and mannose receptor (MR). Expression of mRNA encoding TNF $\alpha$, IL-1 $\beta$, and IL-6 increased at $24 \mathrm{~h}$ and $48 \mathrm{~h}$ post-MCT treatment, and declined thereafter (Fig. 3A-C). Although the expression of mRNA encoding Fizz1 and MR did not change during the injury phase of MCT-induced hepatotoxicity, expression of Fizz1 increased at $72 \mathrm{~h}$, and that of MR increased at 72, 96, and $120 \mathrm{~h}$ post-treatment (Fig. 3D and E). Thus, increased expression of mRNA encoding markers of reparative macrophages is associated with increased numbers of these macrophages in the liver.

Involvement of SDF-1-CXCR4 in liver repair after $M C T$-induced liver injury. To investigate the role of CXCR4 in the accumulation of macrophages during MCT-induced liver injury, we measured the expression of CXCR4 mRNA in the liver after MCT treatment. Expression of CXCR4 mRNA was upregulated at 48 and $72 \mathrm{~h}$ after MCT treatment (Fig. 4A). Expression of mRNA encoding SDF-1, the ligand 
A

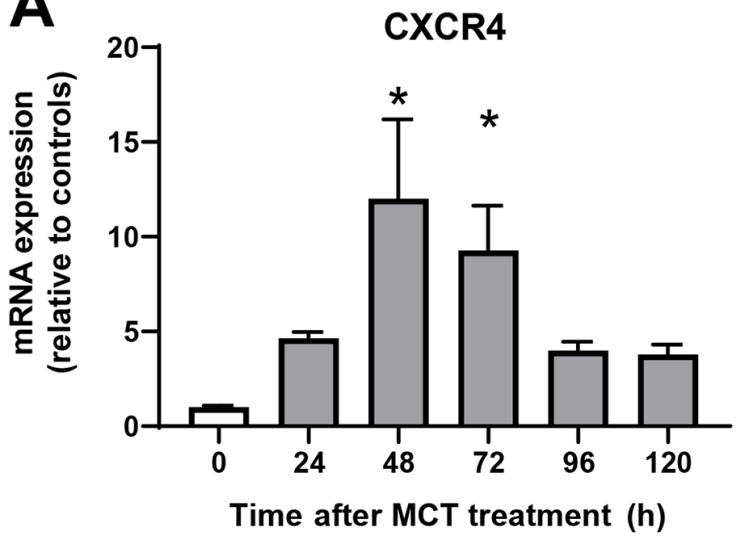

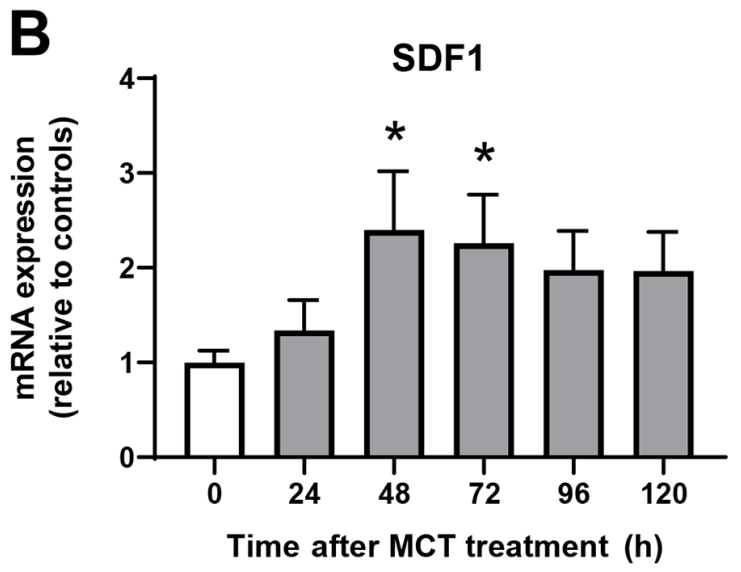

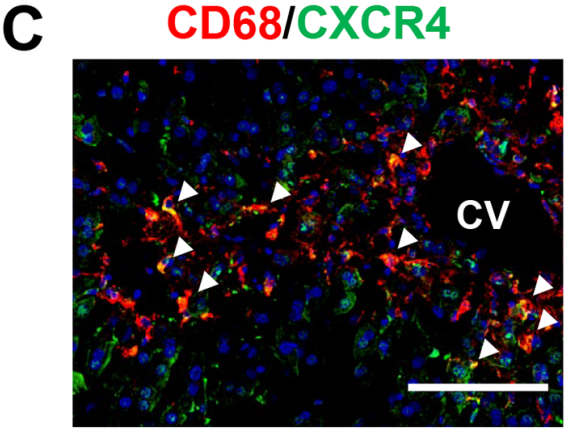

$\mathbf{E}$

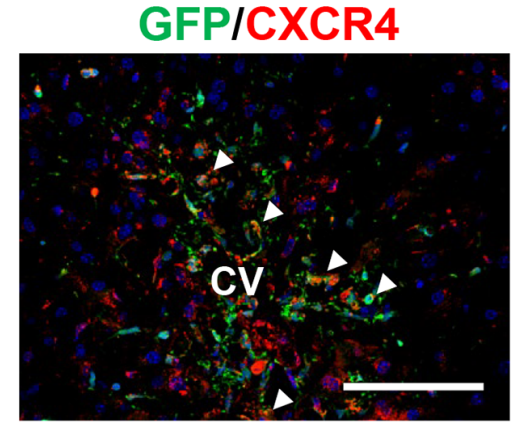

GFP/CD68

D

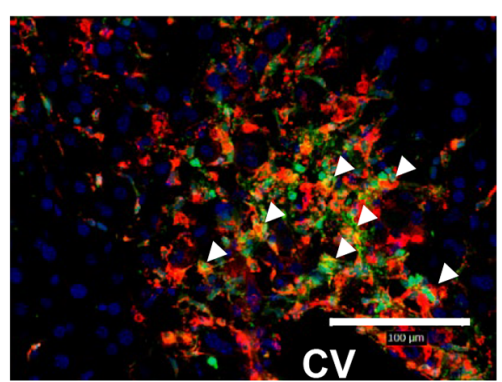

$\mathbf{F}$

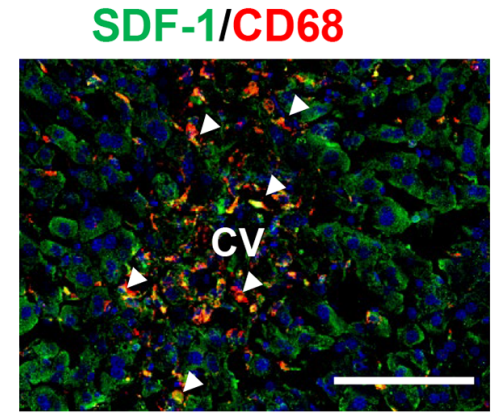

SDF-1/aSMA

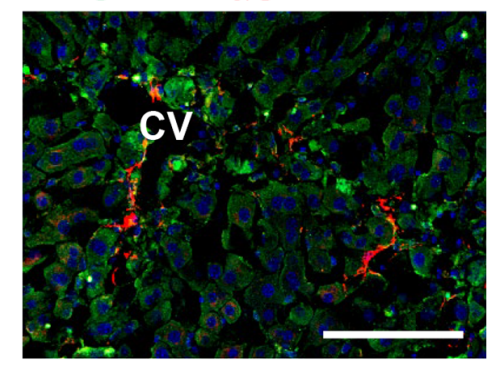

Figure 4. Macrophages expressing CXCR4 accumulate in the liver during the repair phase of MCT hepatotoxicity. Expression of mRNA encoding (A) SDF-1 and (B) CXCR4 in the livers of mice treated with MCT. Data are expressed as the mean \pm SD ( $\mathrm{n}=4-6$ mice per group). $\mathrm{P}<0.05$ vs. 0 h. Representative double immunofluorescence images showing (C) CD68 (red) and CXCR4 (green) or (D) GFP (green) in the livers of mice at $72 \mathrm{~h}$ post-MCT treatment. White triangles indicate merged cells. Scale bars represent $100 \mu \mathrm{m}$. (E) Representative double immunofluorescence images of CXCR4 (red) and GFP (green) in the livers of mice at $72 \mathrm{~h}$ post-MCT treatment. White triangles indicate merged cells. Scale bars represent $100 \mu \mathrm{m}$. (F) Representative double immunofluorescence images of SDF-1 (green) and CD68 (red) or $\alpha$ SMA (red) in the livers of mice at $72 \mathrm{~h}$ post- MCT treatment. CV, central vein. White triangles indicate merged cells. Scale bars, $100 \mu \mathrm{m}$. CXCR4, C-X-C chemokine receptor type 4; MCT, monocrotaline; SDF-1, stromal cell-derived factor-1; GFP, green fluorescent protein; $\alpha$ SMA, $\alpha$ smooth muscle actin; $C V$, central vein.

of CXCR4, also increased at 48 and $72 \mathrm{~h}$ post-MCT treatment (Fig. 4B). To investigate the cellular source of CXCR4, we performed immunofluorescence analysis of CXCR4 expression in liver tissues treated with MCT. Expression of CXCR4 co-localized with $\mathrm{CD}^{+} 8^{+}$cells accumulated in the injured regions at $72 \mathrm{~h}$ after MCT treatment (Fig. 4C), indicating that macrophages are the main source of CXCR4. We also examined whether the accumulated macrophages were derived from the BM. Immunofluorescence analyses of $\mathrm{GFP}^{+} \mathrm{BM}$ chimera mice revealed that both $\mathrm{CD}^{+}$ cells and $\mathrm{GFP}^{+}$cells were extensively accumulated in the injured centrilobular regions at $72 \mathrm{~h}$ post-MCT treatment, and that $\mathrm{CD}^{2} 8^{+}$cells partially co-localized with $\mathrm{GFP}^{+}$ cells (Fig. 4D), indicating that at least some of the macrophages were recruited from the BM. In addition, $\mathrm{CXCR}^{+}$ cells accumulated in the centrilobular regions also partly co-stained with $\mathrm{GFP}^{+}$cells in the liver at $72 \mathrm{~h}$ after MCT treatment, indicating that some of the $\mathrm{CXCR}^{+}$cells were derived from the BM (Fig. 4E).

To further investigate the cellular source of intrahepatic SDF-1, we performed immunofluorescence analysis of SDF-1 in liver tissues treated with MCT for $72 \mathrm{~h}$. SDF-1 ${ }^{+}$cells were accumulated around the central vein, and expression of SDF-1 was co-localized with that of CD68, but not with that of $\alpha$ SMA (Fig. 4F), indicating that macrophages, and not hepatic stellate cells, are the main source of SDF-1 during the repair phase of MCT hepatotoxicity.

Blockade of CXCR4 delays liver repair after MCT-induced liver injury. To examine the functional relevance of CXCR4 

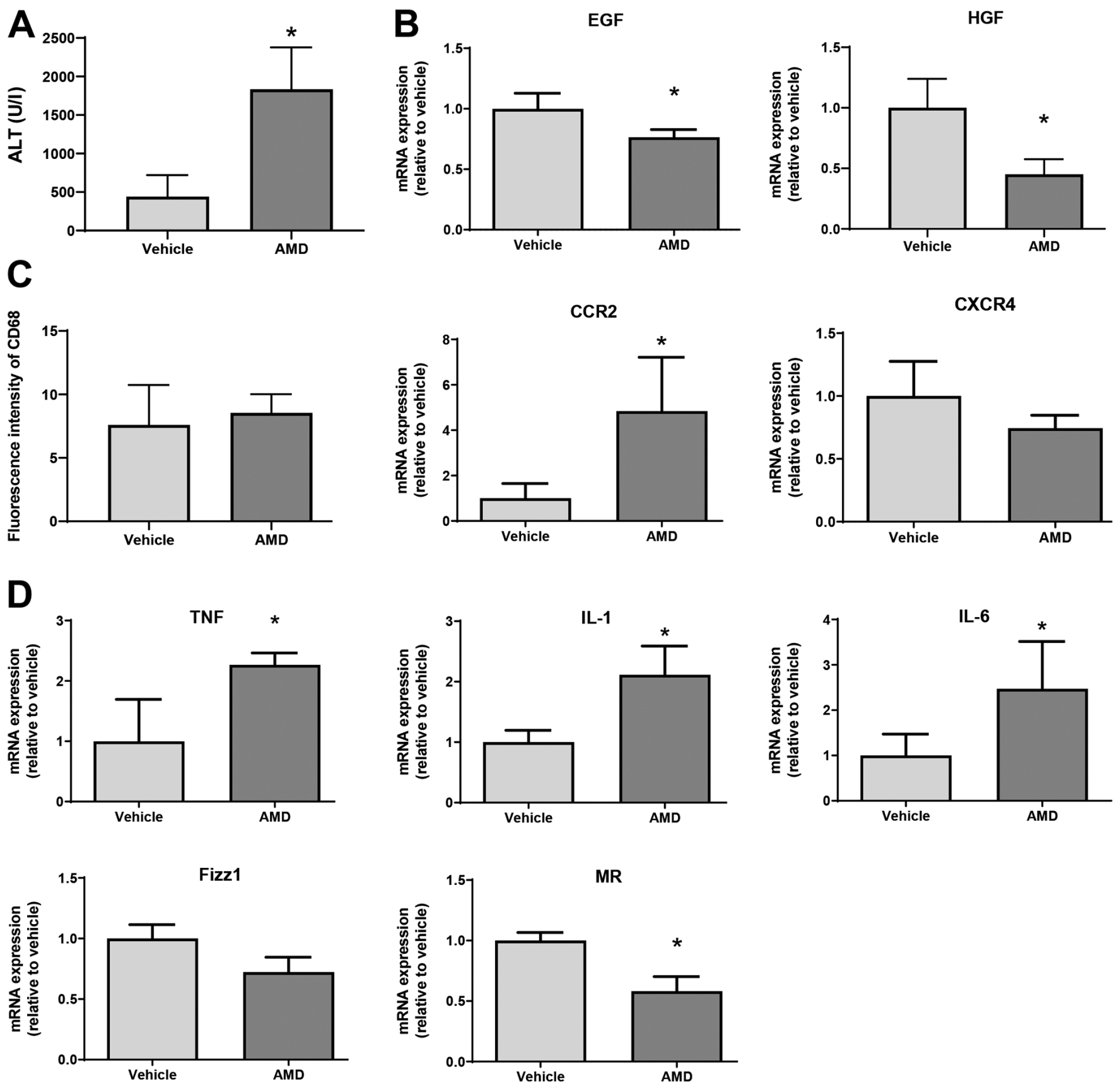

Figure 5. Effect of AMD3100 on liver repair after MCT treatment. (A) ALT levels in mice treated with AMD3100 or vehicle at $72 \mathrm{~h}$ post-MCT treatment (n=4 mice per group). (B) Expression of mRNA encoding HGF and EGF in the livers of mice treated with AMD3100 or vehicle at $72 \mathrm{~h}$ post-MCT treatment ( $\mathrm{n}=3-4$ mice per group). (C) Fluorescence intensity of CD68 and mRNA expression of CCR2 and CXCR4 in the livers of mice treated with AMD3100 or vehicle at 72 $\mathrm{h}$ post-MCT treatment ( $\mathrm{n}=4$ mice per group). (D) Expression of mRNA encoding TNF $\alpha$, IL-1 $\beta$, IL-6, Fizz1 and MR in the livers of mice treated with AMD3100 or vehicle at $72 \mathrm{~h}$ post-MCT treatment ( $\mathrm{n}=4-5$ mice per group). Data are expressed as the mean $\pm \mathrm{SD}$. $\mathrm{P}<0.05$ vs. vehicle. MCT, monocrotaline; ALT, alanine transaminase; HGF, hepatocyte growth factor; EGF, epidermal growth factor; CCR2, C-C motif chemokine receptor 2; CXCR4, C-X-C chemokine receptor type 4; TNF, tumor necrosis factor; IL, interleukin; Fizz1, found in inflammatory zone 1; MR, mannose receptor.

during the repair phase of MCT hepatotoxicity, mice received an i.p. injection of AMD3100, a specific inhibitor of CXCR4. As shown in Fig. 5A, ALT levels at $72 \mathrm{~h}$ in mice treated with AMD3100 were higher than those in mice treated with vehicle. In addition, levels of EGF and HGF mRNA in AMD3100-treated mice were lower at $72 \mathrm{~h}$ post-MCT treatment than those in vehicle-treated mice (Fig. 5B). Although the number of $\mathrm{CD} 68^{+}$cells, as indicated by the fluorescence intensity of CD68 in AMD3100-treated mice was not different from that in vehicle-treated mice, the level of C-C motif chemokine receptor 2 (CCR2) mRNA in AMD3100-treated mice was higher than those in vehicle-treated mice (Fig. 5C). During hepatic inflammation, CCR2 is primarily found in monocyte-derived macrophages, which are characterized as pro-inflammatory macrophages (9). Regarding CXCR4 expression, there was no statistical difference in CXCR4 levels between the two treatments. Furthermore, AMD3100 increased the expression of mRNA encoding markers of pro-inflammatory macrophages (i.e., TNF $\alpha$, IL-1 $\beta$, and IL-6) and decreased the expression of mRNA encoding markers of 

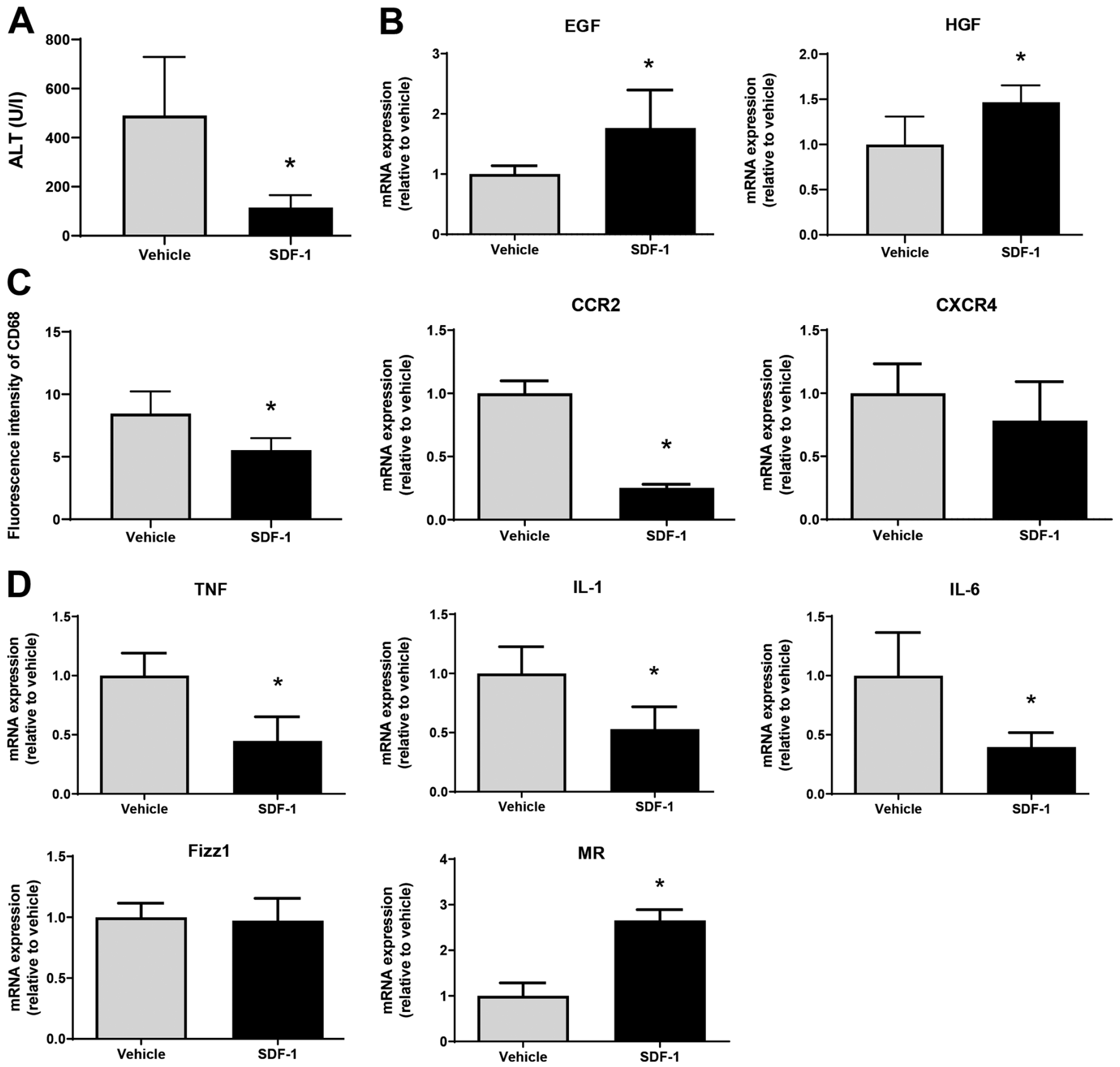

Figure 6. Effects of SDF-1 on liver repair after MCT treatment. (A) ALT levels in mice treated with SDF-1 or vehicle at $72 \mathrm{~h}$ post-MCT treatment (n=4 mice per group). (B) mRNA expression of HGF and EGF in the livers of mice treated with SDF-1 or vehicle at $72 \mathrm{~h}$ post-MCT treatment (n=4-5 mice per group). (C) Fluorescence intensity of CD68 and mRNA expression of CCR 2 and CXCR4 in the livers of mice treated with SDF-1 or vehicle at $72 \mathrm{~h}$ post-MCT treatment ( $\mathrm{n}=4-5$ mice per group). (D) mRNA expression of TNF $\alpha$, IL-1 $\beta$, IL-6, Fizz1 and MR in the livers of mice treated with SDF-1 or vehicle at $72 \mathrm{~h}$ post-MCT treatment ( $\mathrm{n}=4-5$ mice per group). Data are expressed as the mean $\pm \mathrm{SD}$. " $\mathrm{P}<0.05$ vs. vehicle. SDF-1, stromal cell-derived factor-1; MCT, monocrotaline; ALT, alanine transaminase; HGF, hepatocyte growth factor; EGF, epidermal growth factor; CCR2, C-C motif chemokine receptor 2; CXCR4, C-X-C chemokine receptor type 4; TNF, tumor necrosis factor; IL, interleukin; Fizz1, found in inflammatory zone 1; MR, mannose receptor.

reparative macrophages (i.e., MR, but not Fizz1) at $72 \mathrm{~h}$ after MCT treatment (Fig. 5D). These results suggest that CXCR4 plays a critical role in promoting liver repair after MCT administration, and that this repair is associated with a reduction in the expression of genes related to pro-inflammatory macrophage markers.

SDF-1 facilitates liver repair after MCT-induced liver injury. Finally, we examined whether SDF-1 affects liver repair after MCT treatment. SDF-1 reduced ALT levels at $72 \mathrm{~h}$ post-MCT treatment, which was associated with an increased expression of
mRNA encoding EGF and HGF (Fig. 6A and B). The fluorescence intensity of CD68 in the liver of SDF-1-treated mice was lower than that in vehicle-treated mice, which was associated with the downregulation of mRNA encoding CCR2 (Fig. 6C). However, there was no statistical difference in CXCR4 mRNA levels between the two treatments. SDF-1 also decreased the expression of mRNA encoding TNF $\alpha$, IL-1 $\beta$, and IL-6, and increased the expression of mRNA encoding MR (but not Fizz1) (Fig. 6D). These results suggest that SDF-1/CXCR4 plays a critical role in promoting liver repair after MCT administration. 


\section{Discussion}

The objective of this study was to investigate the contribution of macrophages to liver repair after MCT-induced liver injury in a mouse model of SOS. We found that macrophages derived from the BM accumulated in the liver to repair damaged tissue. This was associated with increased hepatic expression of SDF-1 and CXCR4 during the repair phase. The SDF-1-CXCR4 axis plays a role in liver repair by recruiting macrophages with a reparative phenotype.

Initially, ALI induced by MCT was characterized by damage to the LSEC, including gaps in the surface of the LSEC and detachment of the LSEC from the sinusoidal wall $(4,5)$. LSEC injury allows blood components to penetrate into the space of Disse, resulting in centrilobular hepatocellular damage associated with the accumulation of platelets and macrophages (12). Of note, we found that hepatic inflammation induced by MCT administration was resolved and that severe liver damage was repaired. Significant MCT-induced liver injury, as evidenced by increased levels of ALT and hepatic necrosis, were restored to normal levels. This was associated with increased expression of mRNA encoding TNF $\alpha$, IL-1 $\beta$, and IL- 6 during the injury phase of MCT hepatotoxicity and increased expression of mRNA encoding Fizz1 and MR during the repair phase. In addition, expression of mRNA encoding hepatic trophic growth factors HGF and EGF increased during the recovery phase.

Macrophages are key drivers of recovery in liver tissues damaged by acute injury (9) caused by chemicals such as acetaminophen (7) and carbon tetrachloride (8). Upon liver injury induced by MCT, resident Kupffer cells in the injured regions are depleted (4). Macrophages sense liver injury, and monocyte-derived macrophages accumulate at the site to replenish diminished Kupffer cells and repair damaged tissues (9). The results of the current study demonstrate that macrophages accumulate in injured regions during the repair phase of MCT hepatotoxicity, and that accumulated macrophages are, at least in part, derived from the BM. Based on the profiles of proinflammatory and anti-inflammatory mediators in the liver after MCT treatment, reparative macrophages contribute to liver repair from acute MCT-induced liver injury.

It has been reported that, during MCT-induced inflammation, the co-administration of MCT and lipopolysaccharide increases ALT levels at $24 \mathrm{~h}$ post-treatment, which is associated with the upregulation of chemokine (C-X-C motif) ligand 16 (CXCL16) in hepatocytes (23). Inhibition of the SDF-1CXCR4 axis suppresses the development of MCT-induced pulmonary hypertension, which is accompanied by an increase in CXCR4-expressing cells in the BM (20). These results indicate that chemokines and their receptors are involved in the progression of MCT toxicity in the liver and lungs. In addition, the present study demonstrated that SDF-1-CXCR4 pathway contributes to promoting the resolution of hepatic inflammation and liver tissue recovery from acute MCT-induced liver injury. Although C-X-C chemokine receptors are essential for the regulation of immune cell recruitment to sites of inflammatory injury, it remains unknown whether $\mathrm{C}-\mathrm{X}$-C chemokine receptors other than CXCR4 play a role in MCT-mediated inflammation. Further studies are needed to clarify this in MCT-induced liver injury.
During ALI, SDF-1 plays an important role in the regeneration by promoting hepatocyte proliferation. The SDF-1-CXCR4 axis is essential for recruiting stem cells from the BM to sites of injury to promote tissue repair (13). In the liver environment, SDF-1 is produced by biliary epithelial cells, hepatic stellate cells, and LSECs $(9,24,25)$. In this study, immunofluorescence analyses revealed that SDF-1 was expressed by macrophages, but not by hepatic stellate cells. In addition, CXCR4, the specific receptor for SDF-1, was expressed by macrophages, including BM-derived macrophages. Both CXCR4 and SDF-1 were upregulated after MCT treatment and during the repair phase. Pharmacological inhibition of CXCR4 by AMD3100 aggravated MCT-induced liver injury, as evidenced by increased ALT levels and delayed liver repair (indicated by a reduction in hepatic levels of EGF and HGF). Although the number of accumulated macrophages in the liver did not differ between mice treated with AMD3100 and vehicle, increased mRNA levels related to the pro-inflammatory macrophage phenotype (i.e., TNF $\alpha$, IL-1 $\beta$, IL-6, and CCR2), and reduced levels of markers of a reparative macrophage phenotype (i.e., MR), suggesting that AMD3100 may promote accumulation of pro-inflammatory cells. Consistent with this, a previous study showed that CXCR4 blockade in mice inhibits hepatocyte proliferation after acetaminophen treatment, indicating that CXCR4 signaling promotes liver regeneration (18). In addition, AMD3100 leads to sustained hepatic inflammation induced by carbon tetrachloride injection, along with increased hepatic necrosis and increased numbers of infiltrating inflammatory cells (17). Furthermore, there was no statistical difference in CXCR4 levels between AMD3100 and vehicle, indicating that AMD3100 did not change the hepatic expression of CXCR4 at $72 \mathrm{~h}$ after MCT treatment. These results suggest that AMD3100 inhibits CXCR4-mediated signal transduction pathways (26) without affecting the expression of CXCR4 in the livers of mice treated with MCT.

In contrast, administration of SDF-1 accelerated liver repair after MCT-induced ALI, which was associated with the downregulation of genes related to a pro-inflammatory macrophage phenotype and upregulation of genes related to a reparative macrophage phenotype. SDF-1 administration also decreased the expression of CCR2, which is primarily found in pro-inflammatory macrophages (22), suggesting that SDF-1 reduced the accumulation of $\mathrm{CD} 68^{+}$-cells characterized by pro-inflammatory macrophages. These results suggest that SDF-1 may be a key driver of liver repair after MCT administration. Previous studies suggest that binding of SDF-1 to CXCR4 plays a role in liver repair by recruiting hematopoietic stem cells to the site of injury in the liver (19). In addition, mice treated with a CXCR4 inhibitor are more susceptible to chronic liver injury (17). Similarly, upregulation of SDF-1 in the liver after partial hepatectomy increases mobilization of LSEC precursors from the BM to promote liver regeneration in rats (27). Taken together, these findings, including our own, indicate an important role for the SDF-1/CXCR4 axis in liver repair and regeneration in response to chemical-induced acute or chronic injury or partial hepatectomy.

Because SDF-1 recruits macrophages through CXCR4 signaling, the administration of SDF-1 would upregulate CXCR4 expression in livers; however, the results showed that the levels of CXCR4 expression in SDF-1-treated mice did not 
differ from those in vehicle-treated mice. The current study demonstrated that SDF-1 treatment attenuated liver injury at $72 \mathrm{~h}$ after MCT treatment, which was associated with reduced accumulation of macrophages in the liver. Thus, it is speculated that SDF-1 administration did not upregulate CXCR4 expression at $72 \mathrm{~h}$ after MCT treatment.

However, others report that administration of recombinant SDF-1 decreases the proliferation of hepatocytes after hepatic I/R injury in mice (28). Furthermore, they found that treatment of mice with AMD3100 resulted in increased hepatocyte proliferation and reduced necrosis after hepatic I/R. These results imply that the SDF-1-CXCR4 axis suppresses liver repair after hepatic I/R. Such discrepancy may be due to the different models and the different experimental protocols employed for pharmacological intervention.

In the current study, enhanced liver repair from MCT hepatotoxicity was associated with increased hepatic levels of EGF and HGF. Previously, we showed that EGF in macrophages plays a critical role in facilitating liver repair after ALI induced by hepatic I/R (29). Upregulation of HGF is related to liver recovery from acute chemical-induced liver injury (8). These results suggest that macrophages accumulate and repair tissues damaged by MCT by producing EGF and HGF; however, further studies are needed to confirm this.

In conclusion, this study showed that MCT-induced liver injury is repaired by the accumulation of macrophages in the injured region, and that the SDF-1/CXCR4 axis plays an important role in acute liver injury repair by recruiting reparative macrophages. Thus, SDF-1 may be useful for improving recovery from MCT-induced ALI.

\section{Acknowledgements}

The authors would like to thank Ms. Michiko Ogino and Ms. Kyoko Yoshikawa (Department of Pharmacology, Kitasato University School of Medicine, Sagamihara, Kanagawa 252-0374, Japan) for their technical assistance.

\section{Funding}

This work was supported by grants from the Japanese Ministry of Education, Culture, Sports, Science and Technology (MEXT; grant nos. 19K09156 and 20K17630). This study was also supported by the Takeda Science Foundation.

\section{Availability of data and materials}

The datasets used and/or analyzed during the current study are available from the corresponding author on reasonable request.

\section{Authors' contributions}

FO and YI conceived, designed and performed the experiments, and wrote the manuscript. SN, NN and TH performed the experiments. $\mathrm{KH}$ and $\mathrm{MM}$ performed data analysis and interpretation, and provided technical support. WK and HA performed data analysis and interpretation and revised the manuscript critically for intellectual content. FO and YI confirm the authenticity of all the raw data. All authors have read and approved the final manuscript.

\section{Ethics approval and consent to participate}

All experimental procedures were approved by the Animal Experimentation and Ethics Committee of the Kitasato University School of Medicine (permit no. 2019-036, 2020-103).

\section{Patient consent for publication}

Not applicable.

\section{Competing interests}

The authors declare that they have no competing interests.

\section{References}

1. DeLeve LD, Shulman HM and McDonald GB: Toxic injury to hepatic sinusoids: Sinusoidal obstruction syndrome (venoocclusive disease). Semin Liver Dis 22: 27-42, 2002.

2. Coppell JA, Richardson PG, Soiffer R, Martin PL, Kernan NA, Chen A, Guinan E, Vogelsang G, Krishnan A, Giralt S, et al: Hepatic veno-occlusive disease following stem cell transplantation: Incidence, clinical course, and outcome. Biol Blood Marrow Transplant 16: 157-168, 2010.

3. Valla DC and Cazals-Hatem D: Sinusoidal obstruction syndrome. Clin Res Hepatol Gastroenterol 40: 378-385, 2016.

4. DeLeve LD, McCuskey RS, Wang X, Hu L, McCuskey MK, Epstein RB and Kanel GC: Characterization of a reproducible rat model of hepatic veno-occlusive disease. Hepatology 29: 1779-1791, 1999.

5. DeLeve LD, Ito Y, Bethea NW, McCuskey MK, Wang X and McCuskey RS: Embolization by sinusoidal lining cells obstructs the microcirculation in rat sinusoidal obstruction syndrome. Am J Physiol Gastrointest Liver Physiol 284: G1045-G1052, 2003.

6. Mehendale HM: Tissue repair: An important determinant of final outcome of toxicant-induced injury. Toxicol Pathol 33: 41-51, 2005

7. Kato T, Ito Y, Hosono K, Suzuki T, Tamaki H, Minamino T, Kato S, Sakagami H, Shibuya M and Majima M: Vascular endothelial growth factor receptor-1 signaling promotes liver repair through restoration of liver microvasculature after acetaminophen hepatotoxicity. Toxicol Sci 120: 218-229, 2011.

8. Minamino T, Ito Y, Ohkubo H, Hosono K, Suzuki T, Sato T, Ae T, Shibuya A, Sakagami H, Narumiya S, et al: Thromboxane $\mathrm{A}(2)$ receptor signaling promotes liver tissue repair after toxic injury through the enhancement of macrophage recruitment. Toxicol Appl Pharmacol 259: 104-114, 2012.

9. Krenkel O and Tacke F: Liver macrophages in tissue homeostasis and disease. Nat Rev Immunol 17: 306-321, 2017.

10. Nishizawa N, Ito Y, Eshima K, Ohkubo H, Kojo K, Inoue T, Raouf J, Jakobsson PJ, Uematsu S, Akira S, et al: Inhibition of microsomal prostaglandin E synthase-1 facilitates liver repair after hepatic injury in mice. J Hepatol 69: 110-120, 2018.

11. Nakamoto S, Ito Y, Nishizawa N, Goto T, Kojo K, Kumamoto Y, Watanabe M, Narumiya S and Majima M: EP3 signaling in dendritic cells promotes liver repair by inducing IL-13-mediated macrophage differentiation in mice. FASEB J 34: 5610-5627, 2020.

12. Otaka F, Ito Y, Inoue T, Ohkubo H, Nishizawa N, Kojo K, Betto T, Yamane S, Narumiya S, Koizumi W, et al: Thromboxane A2 receptor signaling in endothelial cells attenuates monocrotalineinduced liver injury. Toxicol Appl Pharmacol 381: 114733, 2019.

13. Liepelt A and Tacke F: Stromal cell-derived factor-1 (SDF-1) as a target in liver diseases. Am J Physiol Gastrointest Liver Physiol 311: G203-G209, 2016.

14. Jin DK, Shido K, Kopp HG, Petit I, Shmelkov SV, Young LM, Hooper AT, Amano H, Avecilla ST, Heissig B, et al: Cytokinemediated deployment of SDF-1 induces revascularization through recruitment of $\mathrm{CXCR}^{+}{ }^{+}$hemangiocytes. Nat Med 12: 557-567, 2006.

15. Amano H, Kato S, Ito Y, Eshima K, Ogawa F, Takahashi R, Sekiguchi K, Tamaki H, Sakagami H, Shibuya M, et al: The role of vascular endothelial growth factor receptor-1 signaling in the recovery from ischemia. PLoS One 10: e0131445, 2015. 
16. Sato T, Amano H, Ito Y, Eshima K, Minamino T, Ae T, Katada C, Ohno T, Hosono K, Suzuki T, et al: Vascular endothelial growth factor receptor 1 signaling facilitates gastric ulcer healing and angiogenesis through the upregulation of epidermal growth factor expression on VEGFR $1^{+} \mathrm{CXCR} 4^{+}$cells recruited from bone marrow. J Gastroenterol 49: 455-469, 2014.

17. Saiman Y, Jiao J, Fiel MI, Friedman SL, Aloman C and Bansal MB: Inhibition of the CXCL12/CXCR4 chemokine axis with AMD3100, a CXCR4 small molecule inhibitor, worsens murine hepatic injury. Hepatol Res 45: 794-803, 2015.

18. Tirone M, Tran NL, Ceriotti C, Gorzanelli A, Canepari M, Bottinelli R, Raucci A, Di Maggio S, Santiago C,Mellado M, et al: High mobility group box 1 orchestrates tissue regeneration via CXCR4. J Exp Med 215: 303-318, 2018.

19. Kollet O, Shivtiel S, Chen YQ, Suriawinata J, Thung SN Dabeva MD, Kahn J, Spiegel A, Dar A, Samira S, et al: HGF, SDF-1, and MMP-9 are involved in stress-induced human CD34 stem cell recruitment to the liver. J Clin Invest 112: 160-169, 2003 .

20. Zhang T, Kawaguchi N, Tsuji K, Hayama E, Furutani Y Sugiyama $\mathrm{H}$ and Nakanishi T: Silibinin upregulates CXCR4 expression in cultured bone marrow cells (BMCs) especially in pulmonary arterial hypertension rat model. Cells 9: 1276, 2020.

21. Ogawa F, Amano H, Eshima K, Ito Y, Matsui Y, Hosono K Kitasato H, Iyoda A, Iwabuchi K, Kumagai Y, et al: Prostanoid induces premetastatic niche in regional lymph nodes. J Clin Invest 124: 4882-4894, 2014.

22. Amano H, Mastui Y, Ito Y, Shibata Y, Betto T, Eshima K, Ogawa F, Satoh Y, Shibuya M and Majima M: The role of vascular endothelial growth factor receptor 1 tyrosine kinase signaling in bleomycin-induced pulmonary fibrosis. Biomed Pharmacother 117: 109067, 2019.
23. Hammad MA, Abdel-Bakky MS, Walker LA and Ashfaq MK: Oxidized low-density lipoprotein and tissue factor are involved in monocrotaline/lipopolysaccharide-induced hepatotoxicity. Arch Toxicol 85: 1079-1089, 2011.

24. Hong F, Tuyama A, Lee TF, Loke J, Agarwal R, Cheng X, Garg A, Fiel MI, Schwartz M, Walewski J, et al: Hepatic stellate cells express functional CXCR4: Role in stromal cell-derived factor-1alpha-mediated stellate cell activation. Hepatology 49: 2055-2067, 2009.

25. Sawitza I, Kordes C, Reister S and Häussinger D: The niche of stellate cells within rat liver. Hepatology 50: 1617-1624, 2009

26. Hatse S, Princen K, Bridger G, De Clercq E and Schols D: Chemokine receptor inhibition by AMD3100 is strictly confined to CXCR4. FEBS Lett 527: 255-262, 2002.

27. DeLeve LD, Wang X and Wang L: VEGF-sdf1 recruitment of $\mathrm{CXCR} 7^{+}$bone marrow progenitors of liver sinusoidal endothelial cells promotes rat liver regeneration. Am J Physiol Gastrointest Liver Physiol 310: G739-G746, 2016.

28. Wilson GC, Freeman CM, Kuethe JW, Quillin RC III, Nojima H, Schuster R, Blanchard J, Edwards MJ, Caldwell CC and Lentsch $\mathrm{AB}$ : CXC chemokine receptor-4 signaling limits hepatocyte proliferation after hepatic ischemia-reperfusion in mice. Am J Physiol Gastrointest Liver Physiol 308: G702-709, 2015.

29. Ohkubo H, Ito Y, Minamino T, Eshima K, Kojo K, Okizaki S, Hirata M, Shibuya M, Watanabe M and Majima M: VEGFR1positive macrophages facilitate liver repair and sinusoidal reconstruction after hepatic ischemia/reperfusion injury. PLoS One 9: e105533, 2014

This work is licensed under a Creative Commons Attribution-NonCommercial-NoDerivatives 4.0 International (CC BY-NC-ND 4.0) License. 